Macedonian Pharmaceutical Bulletin, 66 (Suppl 1) 43- 44 (2020)

Online ISSN 1857 - 8969

UDC: $612.379: 577.115 .3]: 159.92$

DOI: 10.33320/maced.pharm.bull.2020.66.03.021

Short communication

\title{
The role of omega-3 for improvement of mood, behavior and communication skills in children
}

\author{
Elena P. Stanojevska ${ }^{1}$, Roberta Mitrevska ${ }^{2}$ \\ ${ }^{I}$ Nobel Ilac Representative - Skopje, St. 8-th Udarna Brigada No. 39/2, 1000 Skopje, N. Macedonia \\ ${ }^{2}$ Association for the right of children end youth with special needs "Lastovica", \\ Blvd. Asnom No. 60-2/18, 1000 Skopje, N. Macedonia
}

\section{Introduction}

The long chain, polyunsaturated fatty acids (LCPUFAs) are well known for more than 100 years. Nowadays, there is a big emphasis on the use of omega-3 and its role for numerous functions in the body. There are two types of PUFAs, omega-6s, which are found primarily in vegetable oils such as sunflower, corn, flaxseed and canola oils, and omega-3s, specifically DHA (docosahexaenoic acid) and EPA (eicosapentaenoic acid), two long-chain PUFAs found primarily in fatty fish, and short-chain ALA (alpha-linolenic acid) that comes primarily from plant-based sources like flax. They are crucial for growing infants and critical for development of the brain and central nervous system. DHA is proven essential to pre- and postnatal brain development, whereas EPA seems more influential on behavior and mood. Both, DHA and EPA generate neuroprotective metabolites (Kidd, 2007).

Adequate dietary availability of DHA and EPA is fundamental to brain function. DHA/EPA are important throughout adulthood, as well as during the brain growth spurts that characterize pre-natal and post natal development (Kidd, 2007).

\section{Omega-3 acids in childhood brain development}

With over 30000 scientific papers published, omega-3 fatty acids are the most studied nutrients in the world. Many people around the world does not consume enough EPA and DHA for brain and heart health support (Murrphy et al., 2015). In 2012, the European Commission authorized an Article 13.1 health claim that min $250 \mathrm{mg}$ per day of EPA and DHA contributes to the maintenance of normal function of the heart, and $250 \mathrm{mg}$ DHA per day contributes for the maintenance of normal brain and eye development. Two-thirds of the human brain is made of fat and DHA as primary structural membrane component makes up to $97 \%$ in the brain and $93 \%$ in the eyes. The fundamental importance of DHA for brain development is beyond despute (McCann and Ames, 2005).

Omega-3 vs. omega-6 fatty acids - understanding the difference

Our body does not produce omega 3 and/or omega 6; therefore, we should get them throughout food.

Omega 6 is very much present in today's modern diet contrary to omega 3 . The ideal ratio of $\omega-3$ vs. $\omega-6$ is 1:2. Maximum accepted is $1: 4$. Nowadays, this ratio goes up to 1:20. Omega 6 fatty acids possess proinflamatory activity, while contrary to this, both DHA and EPA, inhibit the formation of leukotrienes and prostaglandins from arachidonic

* elena.stanojevska@nobel.com.mk 
acid, and $\omega-6$ fatty acid and reduces the generationof cytokines from inflamatory cells (Lee et al., 1985).

DHA/EPA proper daily intake may give positive results for improvement of mood, behavior and communication skills in chlidren

Despite the higher concentration of DHA and EPA needed for more effective therapy for this particular impairment, also the ratio between these two structural similar fatty acids is important as they compete in the gut for absorption. Therefore, the recommended ratio for maximum bioavailability is 1.5:1 for EPA/DHA.

\section{Materials and methods}

\section{Materials}

80 children of age from 17 months till 10 years were supplemented with NBL Fish oil (product of NOBEL ILAC -Turkey) for 6 weeks. NBL fish oil is a high-quality purified omega- 3 product abundant in EPA and DHA.

\section{Methods}

Participants were divided in two groups regarding the age. Children of age from 1-4 were given $5 \mathrm{~mL}$ per day, while children above 4 years 10 $\mathrm{mL}$. The quantity of $10 \mathrm{~mL}$ provides $820 \mathrm{mg}$ of omega 3, out of it $390 \mathrm{mg}$ EPA and $260 \mathrm{mg}$ DHA. The younger group received half concentrations of the daily recommended dosage, respectively for DHA and EPA. A team of specialists were ennroled to conduct a careful assessment of the effects of the product on children's mood, behavior and performances of the communicaition skills (attention, eye contact, focus, social response).

\section{Results and discussion}

What triggered our attention was the positive reaction from both parents and health providers in the association during consuming this omega 3 product. The health professionalists pracitice usage of omega 3 as part of children's treatment for more than 3 years. The omega 3 product had positive influence mainly on the communication skills (shortterm possibility for eye contact, managing of the hyperactivity, keeping focus and longer concentraction). We are aware that the 6 week supplementation period is very short for a conclusion, but the noted results deserve a chance for longer and further investigation, esspecially that this is very sensitive, and more offen present condition nowadays.

\section{Conclusion}

There are so many clinical papers that claim the beneficial effects of omega 3 , but only few explain the role of concentrantion of DHA/EPA and their ratio as key factors for postive effects.

Research into omega-3 fatty acids as a possible treatment for mood, behavior and improvement of the communication and social skills is new and interesting area of research that should be seriously taken into consideration.

The conclusions are that higher concentrations of EPA and DHA are necessary. Moreover, consumers should pay attention to the origin of the fish oil, the fish source and the ratio of DHA/EPA for higher efficacy of the treatment. The daily intake of omega $6 v s$. omega 3 should be as lower as possible and tending to reach ratio of 2:1.

\section{References}

Kidd, M.P., 2007. Omega-3 DHA and EPA for cognition, behavior, and mood: Clinical findings and structural functional synergies with cell membrane phospholipids. Alternative Medicine Review 12(3), 207-227.

Lee, T.H., Hoover, R.I., Williams, J.D., Sperling, R.I., Ravalese 3rd, R., Spur, B.V., Robinson, D.R., Corey, E.J., Lewis, R.A., Austen, K.F., 1985. Effect of dietary enrichment with eicosaentanoic and dohosahexaenoic acid on in-vitro neutrophil and monocyte leucotiene generation and neutrophil funtion. N. Engl. J. Med 312(19), 1217-1224.

McCann, J.C., Ames, B.N., 2005. Is docosahexanoic acid, an n-3long-chain polyunsaturated fatty acid, required for development of normal brain function? An overview of evidence from cognitive and behavioral tests in human and animals. Am. J. Clin. Nutr. 82(2), 281-295.

Murphy, R.A., Yu, E.A., Ciappio, E.D., Mehta, S., McBurney, M.I., 2015. Suboptimal plasma long chain n-3 concentrations are common among adults in the United States, NHANES 2003-2004. Nutrients 7(12), 10282-10289.

Maced. Pharm. Bull. 66 (Suppl 1) 43- 44 (2020) 